\title{
Does the Number of Lymph Nodes Removed During Radical Prostatectomy Impact Risk of Biochemical Recurrence in Patients With Isolated Seminal Vesicle Invasion?
}

\author{
Michael J. Whalen*a, Mark Silva ${ }^{b}$, Michael Rothberg ${ }^{b}$, Edan Y. Shapiro ${ }^{b}$, \\ Trushar Patel ${ }^{c}$, Ari Bergman ${ }^{d}$, ArindamRoyChoudhury ${ }^{\mathrm{e}}$, James McKiernan ${ }^{\mathrm{b}}$, \\ Mitchell C. Benson ${ }^{b}$, Ketan K. Badanif \\ ${ }^{a}$ Department of Urology, Yale New Haven Hospital \\ ${ }^{b}$ Department of Urology, Columbia University College of Physicians and Surgeons \\ ${ }^{c}$ Department of Urology, Morsani College of Medicine, University of South Florida \\ ${ }^{\mathrm{d}}$ Department of Urology, State University of New York Downstate Medical Center \\ ${ }^{e}$ Department of Biostatistics, Columbia University Mailman School of Public Health \\ ${ }^{\mathrm{f}}$ Department of Urology, Icahn School of Medicine at Mount Sinai Hospital \\ *Mjw2117@gmail.com
}

\begin{abstract}
:
Objectives: To determine if greater lymph node yield (LNY) during radical prostatectomy is associated with improved biochemical recurrence for men with prostate cancer and isolated seminal vesicle invasion (SVI). Previous studies have shown that total number of lymph nodes removed at RP results in improved survival, regardless of node positivity, but this has not been examined in men with isolated SVI.

Methods: The IRB-approved Columbia University Urologic Oncology Database was reviewed to identify patients who underwent RP from 1990-2011 and had prostate cancer with isolated SVI (i.e. pT3bNOMx). BCR was defined as two postoperative PSA values $>0.2 \mathrm{ng} / \mathrm{mL}$. Cox proportional hazards model was used to determine if LNY (continuous) was predictive of BCR, when controlling for clinical and pathologic features.

Results: We identified 155 patients with isolated SVI. At a mean follow-up of 48 months, 96 patients (62.5\%) experienced BCR. Estimated 5-year freedom from BCR rate was 45\%. Mean number of LNs removed was 8.9 (1-35). Using the Cox proportional Hazards model, continuous LNY was not significantly associated with risk of $B C R(p=0.25)$, while PSA (HR 2.3, $p=0.01)$, pathologic Gleason score $(p=0.023)$, and bladder neck invasion $(p=0.04)$ were significant.

Conclusion: Seminal vesicle invasion after radical prostatectomy is associated with a high risk of BCR, even in the absence of positive surgical margins or lymphatic involvement. Our study demonstrates that the lymph node yield at the time of prostatectomy does not affect the risk of BCR in men with node-negative SVI. Further studies are warranted to evaluate cancer-specific and overall survival.
\end{abstract}

Keywords: pelvic lymphadenectomy, lymph node yield, advanced prostate cancer, biochemical recurrence

\section{INTRODUCTION}

The finding of seminal vesical invasion (SVI) at the time of radical prostatectomy (RP) is known to be a poor prognostic indicator and is independently associated with BCR and prostate cancer-specific mortality. ${ }^{1-4}$ In additionpatients with SVI at time of RP are more likely to have other poor pathologic features, such as extracapsular extension. ${ }^{5}$ Since patients with this pathologic finding are closely followed and often offered adjuvant therapy, any modification in surgical technique that may reduce the risk of BCR in patients with SVI would be of great importance.

However, patients with SVI represent a heterogeneous group, as demonstrated by the wide range of reports BCR rates for this population. ${ }^{2-4}$ Furthermore, studies have shown that patients with isolated SVI, without nodal involvement (N0) or a positive surgical margin, are at a reduced risk of BCR relative to patients with SVI and nodal involvement (N1). ${ }^{6}$ Whilepatients with a constellation of highrisk features are most likely to benefit from adjuvant therapy due to their increased risk of BCR, those with N0 disease may be cured with RP alone. Yet, even in the absence of histologically discovered positive nodes, a subset of patients will have occult evidence of nodal metastases. Therefore, there 
may be a therapeutic benefit to performing a pelvic lymphadenectomy (PLND) at the time of RP in an effort to treat this micrometastatic disease. While several studies have demonstrated that an increased number of negative normal lymph nodes removed at RP could improve BCR-free survival, this has never been evaluated in a subset of patients with node-negativeisolated SVI. ${ }^{8,9}$

We reviewed our institutional radical prostatectomy experience to explore whether the number of lymph nodes removed at time of surgery impacts the risk of BCR in men with node-negative SVI.

\section{MAterials AND Methods}

\subsection{Patients}

TheColumbia University Urologic Oncology patient database was retrospectively reviewed to identify all patients who underwent radical prostatectomy as definitive treatment for prostate cancer between1998-2010. Informed consent was obtained in accordance with the Declaration of Helsinki and our institutional IRB. Only patients with tissue confirmed adenocarcinoma of the prostate were included in the analysis. Patients who underwent neoadjuvant chemo- or radiotherapy, or those with evidence of metastatic disease at time of initial presentation were excluded. Patients with incomplete pathologic records, less than 12 months of follow-up, or those who did not undergo PLND were excluded. Additionally, patients with LN-positive disease were excluded. The remaining patients represent the study cohort. Demographic data and patient characteristics were collected, including age, preoperative PSA, biopsy Gleason score, clinical stage, and D'Amico risk category.

\subsection{Surgical Technique}

Our robotic assisted radical prostatectomy technique is similar to what has been previously described. ${ }^{10}$ Retropubic prostatectomy was performed by standard technique, through an infrapubic midline incision. The surgical technique employed was based on patient preference and/or the surgeon they were referred to. Decision to perform a PLND at time of RP was at the discretion of the treating surgeon, and usually determined by several factors including extent of disease, stage, and risk of LN involvement as predicted by established nomograms.

\subsection{Pathology Review}

Following surgical extirpation of the prostate, all specimens were fixed in formalin, processed per institutional protocol, and evaluated for pathologic review in a central laboratory. The majority of staging was performed according to $19975^{\text {th }}$ edition TNM classification. ${ }^{11}$ Patients reported to have either pT3b or pT3c disease according to that system were included. Similarly, patients who were staged according to the 2002 system were included if they were pT3b. In addition to the TNM staging, pathologic records were reviewed to record the pathologic Gleason grade, number of positive margins, location of positive margins, and presence of lymphovascular invasion.

\subsection{Outcomes}

The primary outcome analyzed was the presence of biochemical recurrence (BCR), which was defined as a postoperative PSA values $>0.2 \mathrm{ng} / \mathrm{mL}$. Patients with evidence of BCR were censored from date of documented recurrence, while patients free of recurrence were censored at date of last follow-up appointment. Rates of adjuvant and salvage radiation therapy were recorded. Patients who were lost to follow, or not actively followed at our tertiary-care referral institution were excluded from the analysis.

\subsection{Statistical Analysis}

The clinicopathologic variables for the two groups were compared using $\chi^{2}$ test for categorical variables and paired student's $t$ tests for continuous non-parametric data. Lymph node yield was analyzed as a continuous variable; in addition, different cut-off values were selected (1-4, 5-9, 10-15, $>15$; and <10 vs. $\geq 10$ ), and these were analyzed categorically.Univariate and multivariate logistic regression analysis was performed to determine the impact of extend of lymphadenectomy technique on BCR, when controlling for the following clinicopathologic features: age, year of surgery (YOS), preoperative PSA level, clinical stage, biopsy Gleason score, pathologic Gleason score, extracapsular extension (ECE), positive margins (PSM), number of PSM, . Risk of BCR was calculated by the Kaplan-Meier method, and freedom-from-recurrence rates were compared using the log-rank test. A p-value $\leq 0.05$ was considered statistically significant. All analyses were performed on Stata 11.0 (StataCorp LP, College Station, TX). 


\section{ReSUlts}

A total of 4,734patients underwent radical prostatectomy at our institution during the study time period, including 188withevidence of SVI on final pathology. Of those with SVI, 155(82\%) had isolated SVI, without evidence of lymph node involvement on final pathologic review. Baseline demographic and clinical factors are listed in Table 1 . Mean age was $63 \pm 0.54$, mean preoperative PSA 14.9 \pm 1.3 . Approximately half of the patients (51.6\%) were D'Amico high risk and $10 \%$ D'Amico low risk. Radical prostatectomy pathology revealed ECE in $62.5 \%$, positive surgical margin in $38.7 \%$ (53\% of which were multifocal positive), bladder neck invasion in 6\%, and bilateral SVI in $48 \%$. Mean lymph node yield was 8.9 (range 1-35) and remained similar among each D'Amico risk classification (low risk mean 8.5, intermediate risk 8.2, and high risk 9.4, $\mathrm{p}>0.05$ ). Regarding additional therapies, only $4 \%$ of patients received adjuvant radiation therapy and $9.7 \%$ received salvage radiation therapy, of whom $40 \%$ received concurrent androgen deprivation. PSM was a predictor of adjuvant therapy on logistic regression ( $\mathrm{p}=0.05$, odds ratio 8.5).

At a mean follow up time of 48 months, 96patients (62.5\%) demonstrated BCR. Calculated 2-,3-, and 5 -year freedom-from-recurrence rates for the 155 patients with isolated SVI were $61 \%, 54 \%$, and $45 \%$, respectively (Figure 1).The Cox proportional hazards model was used to determine predictors of BCR. Significant variables on univariate analysis included year of surgery $(\mathrm{p}=0.01)$, initial PSA $(\mathrm{p}=0.001)$, pathological Gleason score $(\mathrm{p}=0.003)$, extracapsular extension $(\mathrm{p}=0.007)$, bladder neck invasion $(\mathrm{p}=0.002)$, and multifocal positive margin (continuous variable, $\mathrm{p}=0.042$ ). As expected, salvage radiation therapy predicted $\mathrm{BCR}(\mathrm{HR} 2.6, \mathrm{p}=0.001)$. Of note margin status alone was not predictive for these high risk patients $(\mathrm{p}=0.81)$, nor was administration of adjuvant radiation therapy $(n=6$ patients, $\mathrm{p}=0.11)$. Variables that were found to be significant at the univariate level were included in the multivariate analysis. On multivariate analysis, PSA (HR 2.3, p=.001), pathologic Gleason score $(\mathrm{p}=0.023)$, and bladder neck invasion $(\mathrm{p}=0.04)$ were significant predictors of $\mathrm{BCR}$ (Table 2). A total of $28 \%$ of patients had <5 lymph nodes after pelvic lymphadenectomy, $34 \%$ from 5 $9,25 \% 10-15$, and $14 \%>15$ nodes (Table 1). LNY as a continuous variable was not associated with BCR. Additionally, there was no cut-off value of LNs removed that was significantly associated with BCR.

Sub-analysis of patients with PSA $\leq 10 \mathrm{ng} / \mathrm{mL},>10-20 \mathrm{ng} / \mathrm{mL}$, and $>20 \mathrm{ng} / \mathrm{mL}$; Gleason score $\leq 6,7$, and $\geq 8$; and preoperative D'Amico low, low and intermediate, and high risk groups did not demonstrate any significant influence of LNY on BCR. There was a trend toward significance for lower rate of BCR in preoperative D'Amico low risk patients with $\mathrm{LNY}<10$ (HR 0.001, p=0.08). Furthermore, subanalysis for patients with negative SM did not reveal an association between LNY and BCR ( $p>0.05)$. Despite the substantial proportion of patients with bilateral SVI (48\%), subanalysis of unilateral SVI only did not yield significance of LNY on BCR ( $p>0.05)$.

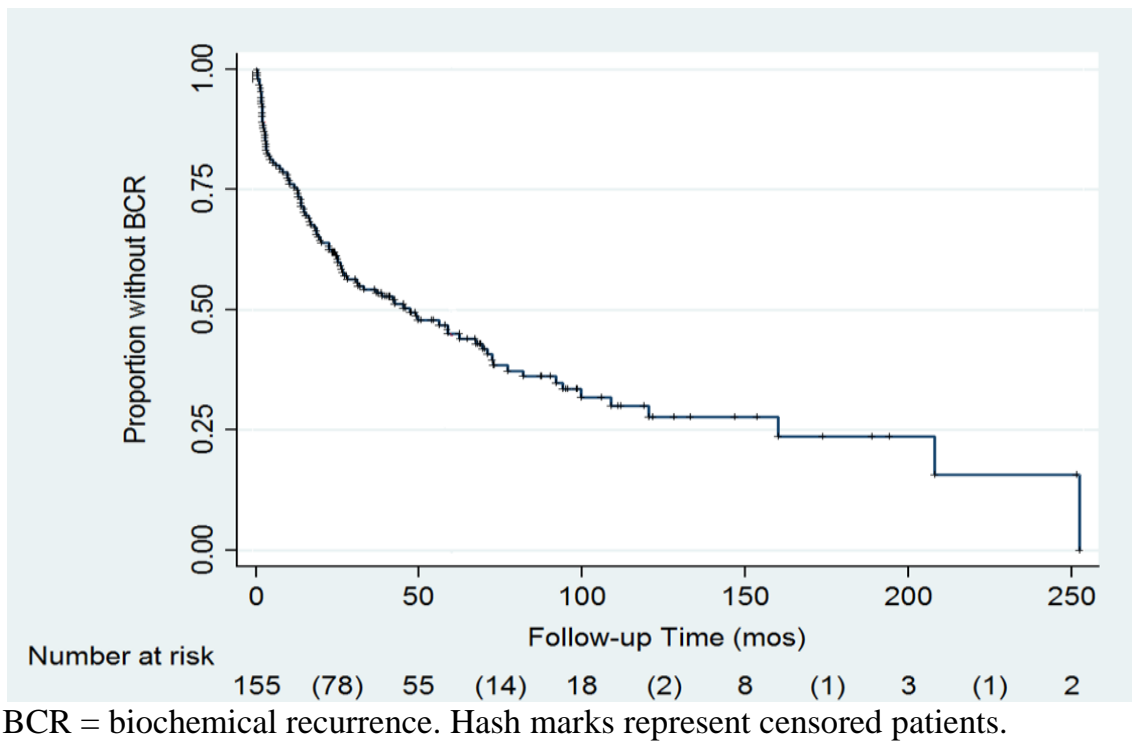

Figure1. Kaplan Meier Curve of Biochemical Recurrence-Free Survival for pT3bNO patients.

$B C R=$ biochemical recurrence. Hash marks represent censored patients. 
Michael J. Whalen et al.

Table1. Patient Demographics. PSA = prostate specific antigen, ECE = extracapsular extension, $S V I=$ seminal vesicle invasion, $L N Y=$ lymph node yield, $X R T=$ radiotherapy; Note that overall total for each clinical stage (i.e. T2) is greater than the sum of each sub-stage (i.e. T2a, T2b, T2c) as some patients' sub-stage was not specified.

\begin{tabular}{|c|c|}
\hline Mean age & $63.3 \pm 0.54$ \\
\hline \multicolumn{2}{|l|}{ Race } \\
\hline White & $85(54.8 \%)$ \\
\hline Black & $32(20.7 \%)$ \\
\hline Hispanic & $17(11 \%)$ \\
\hline Asian & $3(2 \%)$ \\
\hline Other & $17(11 \%)$ \\
\hline Unknown & $1(0.7 \%)$ \\
\hline PSA (mean) & $14.9 \pm 1.3$ \\
\hline \multicolumn{2}{|l|}{ Gleason Score (pathologic) } \\
\hline$\leq 6$ & $12(7.7 \%)$ \\
\hline 7 & $70(45.2 \%)$ \\
\hline 8 & $33(21.3 \%)$ \\
\hline$\geq 9$ & $40(25.8 \%)$ \\
\hline \multicolumn{2}{|l|}{ Clinical Stage } \\
\hline T1c & $82(52.9 \%)$ \\
\hline $\mathrm{T} 2$ & $63(40.6 \%)$ \\
\hline $\mathrm{T} 2 \mathrm{a}$ & $18(11.6 \%)$ \\
\hline $\mathrm{T} 2 \mathrm{~b}$ & $18(11.6 \%)$ \\
\hline $\mathrm{T} 2 \mathrm{c}$ & $17(11 \%)$ \\
\hline T3 & $10(6.4 \%)$ \\
\hline T3a & $1(0.7 \%)$ \\
\hline $\mathrm{T} 3 \mathrm{~b}$ & $3(2 \%)$ \\
\hline $\mathrm{T} 3 \mathrm{c}$ & $2(1.3 \%)$ \\
\hline \multicolumn{2}{|c|}{ D'Amico Risk Group (Clinical) } \\
\hline Low & $15(9.7 \%)$ \\
\hline Intermediate & $60(38.7 \%)$ \\
\hline High & $80(51.6 \%)$ \\
\hline \multicolumn{2}{|l|}{ Pathologic Stage } \\
\hline ECE & $97(62.6 \%)$ \\
\hline Unilateral SVI & $80(51.6 \%)$ \\
\hline Bilateral SVI & $75(48.4 \%)$ \\
\hline Lymph Nodes & $8.9 \pm 0.5(1-35)$ \\
\hline LNY $<5$ & $43(27.7 \%)$ \\
\hline LNY 5-9 & $52(33.6 \%)$ \\
\hline LNY 10-15 & $39(25.2 \%)$ \\
\hline LNY > 15 & $21(13.6 \%)$ \\
\hline Positive Surgical Margin & $60(38.7 \%)$ \\
\hline Adjuvant XRT & $6(3.9 \%)$ \\
\hline Salvage XRT & $15(9.7 \%)$ \\
\hline
\end{tabular}

$P S A=$ prostate specific antigen, ECE = extracapsular extension, $S V I=$ seminal vesicle invasion, $L N Y=l y m p h$ node yield, $X R T$ = radiotherapy; Note that overall total for each clinical stage (i.e. T2) is greater than the sum of each sub-stage (i.e. T2a, T2b, T2c) as some patients' sub-stage was not specified.

Table2. Multivariate Cox Proportional Hazards Model for Biochemical Recurrence. HR = hazard ratio, $S D=$ standard deviation, $Y O S=$ year of surgery, $P S A=$ prostate specific antigen, $G S=$ Gleason score, $E C E=$ extracapsular extension, $B N=$ bladder neck, \# PSM = number of positive surgical margins. $P$-value $\leq 0.05$ is significant.

\begin{tabular}{|c|c|c|c|c|c|}
\hline Variable & HR & SD & P-value & \multicolumn{2}{|c|}{ [95\% Confidence Interval] } \\
\hline YOS & 1.03 & .022 & 0.079 & .995 & 1.08 \\
\hline PSA & $\mathbf{1 . 0 2}$ & .005 & 0.001 & 1.00 & 1.02 \\
\hline PGS & 1.31 & .155 & 0.023 & 1.03 & 1.65 \\
\hline ECE & 1.11 & .159 & 0.445 & .842 & 1.47 \\
\hline BN Invasion & 2.28 & .914 & 0.040 & 1.03 & 5.00 \\
\hline \# PSM & 1.03 & .099 & 0.707 & .859 & 1.25 \\
\hline
\end{tabular}

$H R=$ hazard ratio, $S D=$ standard deviation, $Y O S=$ year of surgery, $P S A=$ prostate specific antigen, $P G S=$ Pathologic Gleason score, ECE = extracapsular extension, BN = bladder neck, \# PSM = number of positive surgical margins. $P$-value $\leq 0.05$ is significant. 


\section{DISCUSSION}

SVI is considered a poor prognostic factor after radical prostatectomy and is associated with increased risk of biochemical failure and disease specific mortality. ${ }^{42-14}$ Since this finding traditionally occurs in men with high volume disease and extracapsular extension, these patients are traditionally offered adjuvant radiotherapy to the prostatic bed and lymph nodes in an attempt to cure the loco-regional spread. However, SVI can also be an isolated finding in the absence of other poor pathologic features. In fact, patients with isolated SVI appear to have improved progression-free rates, relative to those who have additional findings such as regional lymph node involvement and/or positive surgical margins. ${ }^{6}$

Despite their improved outcomes, men with isolated, node-negative SVI continue to experiencehigher BCR rates than those with localized disease. ${ }^{6}$ This suggests that these patients may harbor occult micrometastatic disease, which may be amenable to eradication through a more extensive surgical procedure. In fact, several studies of men undergoing RP have shown that a more extensive LND results in improved oncologic outcomes even if all the nodes removed are negative. ${ }^{8,9}$ The authors of those studies implied that an extensive LND can eliminate undetectable micrometastases or small LN metastases that were missed on routine pathologic examination, and may improve the accuracy of pathological staging.

In contrast, more recent studies have failed to confirm any therapeutic value to an extended LN dissection in node-negative disease. ${ }^{12,13}$ DiMarco et al.found that the extent of lymphadenectomy did not affect the BCR in 7,036 node negative patients with pT1-T3 prostate cancer. ${ }^{12} \mathrm{~A}$ more recent study by Murphy et al. of 964 patients with pT2-4,N0,M0 disease also found that the total number of LN's removed during RP did not decrease the rate of $\mathrm{BCR} .{ }^{13}$ However, these reports included predominately men with low-risk, organ-confined disease. Therefore, these results may not be generalizable to a high-risk population. To our knowledge, no reports have previously examined this population of patients.

To address this question we sought to determine if the number of negative LN's removed impacts the risk of BCR in men known to have node-negative SVI (i.e. isolated pT3b) prostate cancer. On multivariate analysis, we found preoperative PSA value, pathologic Gleason score, and bladder neck invasionto be significantly associated with BCR, consistent with previous reports. However, LN yieldas a continuous variable had no impact on BCR. These findings resemble those of DiMarco et al. and Murphy et al., but more importantly, this is the first report to specifically evaluate this clinical question in a high-risk population of men with isolated SVI. Also, few men in our cohort received adjuvant radiation therapy $(n=9)$, which would enhance the ability to detect an impact on extended lymph node yield on BCR. The fact that adjuvant radiation therapy had no effect on BCR, contrary to recently published randomized data attesting to the overall survival benefit of this therapy for locally advanced prostate cancer, ${ }^{14}$ likely reflects the long time period for our study, with many patients treated prior to the publication of these findings. This observation may also indicate that BCR for pT3b patients reflects systemic recurrence beyond lymph node sites.

It is important to contrast our results to those of Joslyn and Masterson since we failed to find a significant relationship between LN yield and risk of worse oncologic outcomes. ${ }^{8,9}$ First, it is important to distinguish the patient populations. In the report by Masterson et al., 97\% of the patients were <T3, and the vast majority of men in the SEER-based study by Joslyn et al. had clinically localized disease. It is also noteworthy that close to $95 \%$ of the patients in the Joslyn study were white, and these results may not be generalizable to the general population given the known racial disparities in prostate cancer biology. Furthermore, only a fifth of those patients underwent prostatectomy as their initial treatment, thereby introducing potential biases of neoadjuvant radio- or hormonal therapy. In contrast, our study is exclusively made up of patients who underwent RP as their initial therapy and have pathologic T3 disease. In addition to distinguishing the difference in patient population, it is also important to consider that the conclusion of Joslyn et al. that an extended LN dissection improves survival in node negative patients is limited to those who had more than 10 nodes removed; patients with any less than 10 did not have improved survival relative to patients who did not undergo a LN dissection at all. Therefore our use of analyzing $\mathrm{LN}$ yield as a continuous variable is more likely to represent the true significance of $\mathrm{LN}$ dissection. 
It can be concluded from our study that LN yield does not impact BCR in patients with isolated seminal vesicle invasion. This may be partially due to the "Will Rogers" phenomenon. Namely, it is possible that patients who underwent a greater $\mathrm{LN}$ dissection were more likely to have $\mathrm{LN}$ metastases detected, and therefore excluded from our study cohort. This may further help to explain why the LN yield was not found to independently predict BCR rates. A post-hoc analysis of the 22 patients in our database with pT3bN1 disease, however, revealed similar total mean LNY of 8.5 nodes.

The decision to perform extended lymphadenectomy may be based on a patient's high-risk D'Amico category $^{15}$ or an elevated probability $>2-4 \%$ of lymph node involvement on preoperative nomograms. ${ }^{16-17}$ Since we excluded N1 patients from our analysis, our findings cannot really be used to guide the decision of whether or not to perform an extended lymph node dissection. Our findings are useful, however, in the interpretation of the final pathology for pT3bN0 patients vis-à-vis the need for adjuvant radiotherapy. Our results demonstrate that among patients with isolated SVI, being N0 with 2 nodes removed are no more likely to have BCR than being N0 with 15 nodes removed. Although one may postulate that the latter patient is less likely to harbor occult micrometastatic disease and is therefore less likely to occur, our results contradict this intuition. Lymph node yield in pT3bN0 patients should therefore not be used to guide the decision for adjuvant therapy.

This study has several limitations that should be addressed. First, it is a retrospective review, which likely introduces an element of selection bias. Second, it represents a single institution center. Although this is a large, academic, tertiary-care referral center, the results may not be generalizable to all practices. Another limitation is the variable lymph node yield observed across the patients, reflecting inter-surgeon variability in the extent of pelvic lymphadenectomy. Sub-analysis of lymph node yield stratified by D'Amico risk classification, however, did not reveal any differences in mean total LNY among the groups. Thus it appears that preoperative risk category did not influence the extent of lymph node dissection. Perhaps more extensive LND would have to have been performed to detect a BCR difference based on total lymph node yield.

\section{Conclusions}

Seminal vesicle invasion after radical prostatectomy is associated with a high risk of BCR, even in the absence of lymph node involvement. Our study validates that the lymph node yield at the time of prostatectomy does not affect the risk of BCR in men with SVI. Further studies are warranted to evaluate cancer-specific and overall survival.

\section{REFERENCES}

[1] Epstein JI, Carmichael M, Walsh PC. Adenocarcinoma of the prostate invading the seminal vesicle: definition and relation of tumor volume, grade and margins of resection to prognosis. $J$ Urol. May 1993;149(5):1040-1045.

[2] Pound CR, Partin AW, Epstein JI, Walsh PC. Prostate-specific antigen after anatomic radical retropubic prostatectomy. Patterns of recurrence and cancer control. Urol Clin North Am. May 1997;24(2):395-406.

[3] Han M, Partin AW, Pound CR, Epstein JI, Walsh PC. Long-term biochemical disease-free and cancer-specific survival following anatomic radical retropubic prostatectomy. The 15-year Johns Hopkins experience. Urol Clin North Am. Aug 2001;28(3):555-565.

[4] Eggener SE, Scardino PT, Walsh PC, et al. Predicting 15-year prostate cancer specific mortality after radical prostatectomy. J Urol. Mar 2011;185(3):869-875.

[5] Salomon L, Anastasiadis AG, Johnson CW, et al. Seminal vesicle involvement after radical prostatectomy: predicting risk factors for progression. Urology. Aug 2003;62(2):304-309.

[6] Masterson TA, Pettus JA, Middleton RG, Stephenson RA. Isolated seminal vesicle invasion imparts better outcomes after radical retropubic prostatectomy for clinically localized prostate cancer: prognostic stratification of pt3b disease by nodal and margin status. Urology. Jul 2005;66(1):152-155.

[7] Pagliarulo V, Hawes D, Brands FH, et al. Detection of occult lymph node metastases in locally advanced node-negative prostate cancer. J Clin Oncol. Jun 2006;24(18):2735-2742.

[8] Joslyn SA, Konety BR. Impact of extent of lymphadenectomy on survival after radical prostatectomy for prostate cancer. Urology. Jul 2006;68(1):121-125. 
[9] Masterson TA, Bianco FJ, Vickers AJ, et al. The association between total and positive lymph node counts, and disease progression in clinically localized prostate cancer. J Urol. Apr 2006;175(4):1320-1324; discussion 1324-1325.

[10] Menon M, Shrivastava A, Kaul S, et al. Vattikuti Institute prostatectomy: contemporary technique and analysis of results. Eur Urol. Mar 2007;51(3):648-657; discussion 657-648.

[11] Sobin LH, Fleming ID. TNM Classification of Malignant Tumors, fifth edition (1997). Union Internationale Contre le Cancer and the American Joint Committee on Cancer. Cancer. Nov 1997;80(9):1803-1804.

[12] DiMarco DS, Zincke H, Sebo TJ, Slezak J, Bergstralh EJ, Blute ML. The extent of lymphadenectomy for pTXNO prostate cancer does not affect prostate cancer outcome in the prostate specific antigen era. J Urol. Apr 2005;173(4):1121-1125.

[13] Murphy AM, Berkman DS, Desai M, Benson MC, McKiernan JM, Badani KK. The number of negative pelvic lymph nodes removed does not affect the risk of biochemical failure after radical prostatectomy. BJU Int. Jan 2010;105(2):176-179.

[14] Thompson IA, Tangen CM, Paradelo J, et al. Adjuvant radiotherapy for pathologic T3N0M0 prostate cancer significantly reduces risk of metastasis and improves survival: long-term followup of a randomized clinical trial. J Urol, 2009; 181(3): 956-962.

[15] Harbin AC and Eun DD. The role of extended pelvic lymphadenectomy with radical prostatectomy for high-risk prostate cancer. Urol Oncol, 2014; (E-pub ahead of work).

[16] Eifler JB, Feng Z, Lin BM, et al. An updated prostate cancer staging nomogram (Partin tables) based on cases from 2006-2011. BJU Int, 2013; 111(1):22-29.

[17] Gakis G, Boorjian SA, Briganti A, et al. The role of radical prostatectomy and lymph node dissection in lymph-node positive prostate cancer: a systematic review of the literature. Eur Urol, 2014; 66(2): 191-9. 